\title{
Named Entity Recognition with Character-Level Models
}

\author{
Dan Klein $^{1}$ and Joseph Smarr ${ }^{2}$ and Huy Nguyen ${ }^{1}$ and Christopher D. Manning ${ }^{1}$ \\ ${ }^{1}$ Computer Science Dept. \\ Stanford University \\ Stanford, CA 94305-9040 \\ ${ }^{2}$ Symbolic Systems Program \\ Stanford University \\ Stanford, CA 94305-2181 \\ $\{k l e i n, h t n g u y e n$, manning $\}$ ecs.stanford.edu \\ jsmarrestanford.edu
}

\begin{abstract}
We discuss two named-entity recognition models which use characters and character $n$-grams either exclusively or as an important part of their data representation. The first model is a character-level HMM with minimal context information, and the second model is a maximum-entropy conditional markov model with substantially richer context features. Our best model achieves an overall $\mathrm{F}_{1}$ of $86.07 \%$ on the English test data $(92.31 \%$ on the development data). This number represents a $25 \%$ error reduction over the same model without word-internal (substring) features.
\end{abstract}

\section{Introduction}

For most sequence-modeling tasks with word-level evaluation, including named-entity recognition and part-ofspeech tagging, it has seemed natural to use entire words as the basic input features. For example, the classic HMM view of these two tasks is one in which the observations are words and the hidden states encode class labels. However, because of data sparsity, sophisticated unknown word models are generally required for good performance. A common approach is to extract word-internal features from unknown words, for example suffix, capitalization, or punctuation features (Mikheev, 1997, Wacholder et al., 1997, Bikel et al., 1997). One then treats the unknown word as a collection of such features. Having such unknown-word models as an add-on is perhaps a misplaced focus: in these tasks, providing correct behavior on unknown words is typically the key challenge.

Here, we examine the utility of taking character sequences as a primary representation. We present two models in which the basic units are characters and character $n$-grams, instead of words and word phrases. Earlier papers have taken a character-level approach to named entity recognition (NER), notably Cucerzan and
Yarowsky (1999), which used prefix and suffix tries, though to our knowledge incorporating all character $n$ grams is new. In section 2, we discuss a character-level HMM, while in section 3 we discuss a sequence-free maximum-entropy (maxent) classifier which uses $n$-gram substring features. Finally, in section 4 we add additional features to the maxent model, and chain these models into a conditional markov model (CMM), as used for tagging (Ratnaparkhi, 1996) or earlier NER work (Borthwick, 1999).

\section{A Character-Level HMM}

Figure 1 shows a graphical model representation of our character-level HMM. Characters are emitted one at a time, and there is one state per character. Each state's identity depends only on the previous state. Each character's identity depends on both the current state and on the previous $n-1$ characters. In addition to this HMM view, it may also be convenient to think of the local emission models as type-conditional $n$-gram models. Indeed, the character emission model in this section is directly based on the $n$-gram proper-name classification engine described in (Smarr and Manning, 2002). The primary addition is the state-transition chaining, which allows the model to do segmentation as well as classification.

When using character-level models for word-evaluated tasks, one would not want multiple characters inside a single word to receive different labels. This can be avoided in two ways: by explicitly locking state transitions inside words, or by careful choice of transition topology. In our current implementation, we do the latter. Each state is a pair $(t, k)$ where $t$ is an entity type (such as PERSON, and including an other type) and $k$ indicates the length of time the system has been in state $t$. Therefore, a state like (PERSON, 2) indicates the second letter inside a person phrase. The final letter of a phrase is a following space (we insert one if there is none) and the state is a special final state like (PERSON, F). Additionally, once $k$ reaches our $n$-gram history order, it stays there. We then use empirical, unsmoothed estimates for state- 


\begin{tabular}{|l|c|cccc|}
\hline Description & ALL & LOC & MISC & ORG & PER \\
\hline Offi cial Baseline & 71.2 & 80.5 & 83.5 & 66.4 & 55.2 \\
\hline Word-level HMM & 74.5 & 79.5 & 69.7 & 67.5 & 77.6 \\
\hline Char-level, no conx & 82.2 & 86.1 & 82.2 & 73.4 & 84.6 \\
Char-level, context & $\mathbf{8 3 . 2}$ & $\mathbf{8 6 . 9}$ & $\mathbf{8 3 . 0}$ & $\mathbf{7 5 . 1}$ & $\mathbf{8 5 . 6}$ \\
\hline
\end{tabular}

Table 1: $\mathrm{HMM} \mathrm{F}_{1}$ performance, English development set.

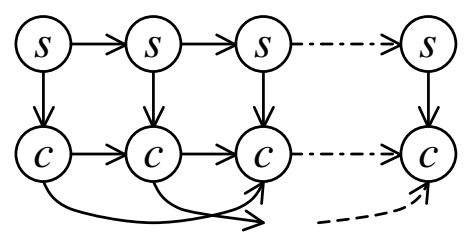

Figure 1: A character-level HMM. The $c$ nodes are character observations and $s$ nodes are entity types.

state transitions. This annotation and estimation enforces consistent labellings in practice. For example, (PERSON, 2) can only transition to the next state (PERSON, 3) or the final state $(P E R S O N, \mathrm{~F})$. Final states can only transition to beginning states, like (other, 1).

For emissions, we must estimate a quantity of the form $\mathrm{P}\left(c_{0} \mid c_{-(n-1)}, \ldots, c_{-1}, s\right)$, for example, $\mathrm{P}(\mathrm{s} \mid$ Thoma, $P E R S O N, 6) .{ }^{1}$ We use an $n$-gram model of order $n=6$. $^{2}$ The $n$-gram estimates are smoothed via deleted interpolation.

Given this model, we can do Viterbi decoding in the standard way. To be clear on what this model does and does not capture, we consider a few examples (_ indicates a space). First, we might be asked for $\mathrm{P}$ (e|to_Denv, $L O C, 5)$. In this case, we know both that we are in the middle of a location that begins with Denv and also that the preceding context was to. In essence, encoding $k$ into the state lets us distinguish the beginnings of phrases, which lets us model trends like named entities (all the classes besides other) generally starting with capital letters in English. Second, we may be asked for quantities like $\mathrm{P}(-\mid$ Italy, $L O C, F)$, which allows us to model the ends of phrases. Here we have a slight complexity: by the notation, one would expect such emissions to have probability 1 , since nothing else can be emitted from a final state. In practice, we have a special stop symbol in our n-gram counts, and the probability of emitting a space from a final state is the probability of the n-gram having chosen the stop character. ${ }^{3}$

\footnotetext{
${ }^{1}$ We index characters, and other vector elements by relative location subscripts: $c_{0}$ is the current character, $c_{1}$ is the following character, and $c_{-1}$ is the previous character.

${ }^{2}$ The smaller space of characters allows us to obtain dense estimates for longer $n$-grams than is possible with word-level models. The value $n=6$ was the empirically optimal order.

${ }^{3}$ This can be cleaned up conceptually by considering the entire process to have been a hierarchical HMM (Fine et al., 1998), where the $n$-gram model generates the entire phrase, followed by a tier pop up to the phrase transition tier.
}

Using this model, we tested two variants, one in which preceding context was discarded (for example, $\mathrm{P}$ (e|to_Denv, $L O C, 5)$ was turned into $\mathrm{P}\left(\mathrm{e} \mid \mathrm{xx}_{-}\right.$Denv, $L O C, 5)$ ), and another where context was used as outlined above. For comparison, we also built a first-order word-level HMM; the results are shown in table 1. We give $F_{1}$ both per-category and overall. The word-level model and the (context disabled) character-level model are intended as a rough minimal pair, in that the only information crossing phrase boundaries was the entity type, isolating the effects of character- vs word-level modeling (a more precise minimal pair is examined in section 3). Switching to the character model raised the overall score greatly, from $74.5 \%$ to $82.2 \%$. On top of this, context helped, but substantially less, bringing the total to $83.2 \%$.

We did also try to incorporate gazetteer information by adding $n$-gram counts from gazetteer entries to the training counts that back the above character emission model. However, this reduced performance (by $2.0 \%$ with context on). The supplied gazetteers appear to have been built from the training data and so do not increase coverage, and provide only a flat distribution of name phrases whose empirical distributions are very spiked.

\section{A Character-Feature Based Classifier}

Given the amount of improvement from using a model backed by character $n$-grams instead of word $n$-grams, the immediate question is whether this benefit is complementary to the benefit from features which have traditionally been of use in word level systems, such as syntactic context features, topic features, and so on.

To test this, we constructed a maxent classifier which locally classifies single words, without modeling the entity type sequences $s .{ }^{4}$ These local classifiers map a feature representation of each word position to entity types, such as PERSON. ${ }^{5}$ We present a hill-climb over feature sets for the English development set data in table 2 . First, we tried only the local word as a feature; the result was that each word was assigned its most common class in the training data. The overall F-score was 52.29\%, well below the official CoNLL baseline of $71.18 \% .{ }^{6}$ We next added $n$-gram features; specifically, we framed each word with special start and end symbols, and then added every contiguous substring to the feature list. Note that this subsumes the entire-word features. Using the substring features alone scored $73.10 \%$, already breaking the

\footnotetext{
${ }^{4}$ The classifi er was trained using conjugate gradient descent, used equal-scale gaussian priors for smoothing, and learned models of over $800 \mathrm{~K}$ features in approximately 2 hours.

${ }^{5}$ The B-/I- distinction in the data was collapsed, though see section 4 .

${ }^{6}$ The latter assigns phrases at once, which is generally superior, but is noticeably worse at multi-word person names, since it cannot synthesize new fi rst-name/last-name pairs.
} 


\begin{tabular}{|l|l|r|rrrr|}
\hline Description & Added Features & ALL & LOC & MISC & ORG & PER \\
\hline Words & $w_{0}$ & 52.29 & 41.03 & 70.18 & 60.43 & 60.14 \\
Official Baseline & - & 71.18 & 80.52 & 83.52 & 66.43 & 55.20 \\
\hline NGrams & $n\left(w_{0}\right)$ & 73.10 & 80.95 & 71.67 & 59.06 & 77.23 \\
Tags & $t_{0}$ & 74.17 & 81.27 & 74.46 & 59.61 & 78.73 \\
\hline Simple Context & $w_{-1}, w_{0}, t_{-1}, t_{1}$ & 82.39 & 87.77 & 82.91 & 70.62 & 85.77 \\
More Context & $\left\langle w_{-1}, w_{0}\right\rangle,\left\langle w_{0}, w_{1}\right\rangle,\left\langle t_{-1}, t_{0}\right\rangle,\left\langle t_{0}, t_{1}\right\rangle$ & 83.09 & 89.13 & 83.51 & 71.31 & 85.89 \\
\hline Simple Sequence & $s_{-1},\left\langle s_{-1}, t_{-1}, t_{0}\right\rangle$ & 85.44 & 90.09 & 80.95 & 76.40 & 89.66 \\
More Sequence & $\left\langle s_{-2}, s_{-1}\right\rangle,\left\langle s_{-2}, s_{-1}, t_{-2}, t_{-1}, t_{0}\right\rangle$ & 87.21 & 90.76 & 81.01 & 81.71 & 90.80 \\
\hline Final & (see text) & $\mathbf{9 2 . 2 7}$ & $\mathbf{9 4 . 3 9}$ & $\mathbf{8 7 . 1 0}$ & $\mathbf{8 8 . 4 4}$ & $\mathbf{9 5 . 4 1}$ \\
\hline
\end{tabular}

Table 2: CMM performance with incrementally added features on the English development set.

the phrase-based CoNLL baseline, though lower than the no-context HMM, which better models the context inside phrases. Adding a current tag feature gave a score of 74.17\%. At this point, the bulk of outstanding errors were plausibly attributable to insufficient context information. Adding even just the previous and next words and tags as (atomic) features raised performance to $82.39 \%$. More complex, joint context features which paired the current word and tag with the previous and next words and tags raised the score further to $83.09 \%$, nearly to the level of the HMM, still without actually having any model of previous classification decisions.

\section{A Character-Based CMM}

In order to include state sequence features, which allow the classifications at various positions to interact, we have to abandon classifying each position independently. Sequence-sensitive features can be included by chaining our local classifiers together and performing joint inference, i.e., by building a conditional markov model (CMM), also known as a maximum entropy markov model (McCallum et al., 2000).

Previous classification decisions are clearly relevant: for example the sequence Grace Road is a single location, not a person's name adjacent to a location (which is the erroneous output of the model in section 3). Adding features representing the previous classification decision $\left(s_{-1}\right)$ raised the score $2.35 \%$ to $85.44 \%$. We found knowing that the previous word was an other wasn't particularly useful without also knowing its part-of-speech (e.g., a preceding preposition might indicate a location). Joint tag-sequence features, along with longer distance sequence and tag-sequence features, gave $87.21 \%$.

The remaining improvements involved a number of other features which directly targetted observed error types. These features included letter type pattern features (for example 20-month would become $\mathrm{d}-\mathrm{x}$ for digitlowercase and Italy would become Xx for mixed case). This improved performance substantially, for example allowing the system to detect ALL CAPS regions. Table 3 shows an example of a local decision for Grace in the context at Grace Road, using all of the features defined to date. Note that the evidence against Grace as a name completely overwhelms the $n$-gram and word preference for PERSON. Other features included secondprevious and second-next words (when the previous or next words were very short) and a marker for capitalized words whose lowercase forms had also been seen. The final system also contained some simple error-driven postprocessing. In particular, repeated sub-elements (usually last names) of multi-word person names were given type PERSON, and a crude heuristic restoration of B- prefixes was performed. In total, this final system had an F-score of $92.31 \%$ on the English development set. Table 4 gives a more detailed breakdown of this score, and also gives the results of this system on the English test set, and both German data sets.

\section{Conclusion}

The primary argument of this paper is that character substrings are a valuable, and, we believe, underexploited source of model features. In an HMM with an admittedly very local sequence model, switching from a word model to a character model gave an error reduction of about $30 \%$. In the final, much richer chained maxent setting, the reduction from the best model minus $n$-gram features to the reported best model was about $25 \%$ - smaller, but still substantial. This paper also again demonstrates how the ease of incorporating features into a discriminative maxent model allows for productive feature engineering. 


\begin{tabular}{|l|llll|}
\hline & PPREV & PREV & CUR & NEXT \\
\hline States & O & O & LOC & LOC \\
\hline Words & morning & at & Grace & Road \\
Tags & NN & IN & NNP & NNP \\
Types & $\mathrm{x}$ & $\mathrm{x}: 2$ & $\mathbf{X x}$ & Xx \\
\hline
\end{tabular}

\begin{tabular}{|c|c|c|c|c|c|}
\hline & $\mathrm{O}$ & LOC & MISC & ORG & PER \\
\hline \multicolumn{6}{|c|}{ WORDS } \\
\hline PWORD:at & -0.18 & 0.94 & -0.31 & 0.28 & -0.73 \\
\hline CWORD:Grace & -0.01 & 0 & 0 & -0.02 & 0.03 \\
\hline NWORD:Road & 0.02 & 0.27 & -0.01 & -0.25 & -0.03 \\
\hline PWORD-CWORD:at-Grace & 0 & 0 & 0 & 0 & 10 \\
\hline CWORD-NWORD:Grace-Road & 0 & 0 & 0 & 0 & 0 \\
\hline \multicolumn{6}{|c|}{ NGRAMS (prefi x/suffi $x$ only here) } \\
\hline$\langle\mathrm{G}$ & -0.57 & -0.04 & 0.26 & -0.04 & 0.45 \\
\hline$\langle\mathrm{Gr}$ & 0.27 & -0.06 & 0.12 & -0.17 & -0.16 \\
\hline$\langle$ Gra & -0.01 & -0.37 & 0.19 & -0.09 & 0.28 \\
\hline$\langle$ Grac & -0.01 & 0 & 0 & -0.02 & 0.03 \\
\hline$\langle$ Grace & -0.01 & 0 & 0 & -0.02 & 0.03 \\
\hline$\langle$ Grace $\rangle$ & -0.01 & 0 & 0 & -0.02 & 0.03 \\
\hline Grace $\rangle$ & -0.01 & 0 & 0 & -0.02 & 0.03 \\
\hline race $\rangle$ & 0 & 0 & 0 & -0.02 & 0.03 \\
\hline ace $\rangle$ & 0.08 & 0.24 & 0.07 & -0.30 & -0.10 \\
\hline ce) & 0.44 & 0.31 & -0.34 & -0.02 & -0.38 \\
\hline e) & 0.38 & -0.14 & -0.18 & -0.06 & 0 \\
\hline \multicolumn{6}{|c|}{ TAGS } \\
\hline PTAG:IN & -0.40 & 0.24 & 0.16 & 0.08 & -0.08 \\
\hline CTAG:NNP & -1.09 & 0.45 & -0.26 & 0.43 & 0.47 \\
\hline NTAG:NNP & 0.05 & -0.19 & 0.18 & -0.12 & 0.08 \\
\hline PTAG-CTAG:IN-NNP & 0 & 0.14 & -0.03 & -0.01 & -0.10 \\
\hline CTAG-NTAG:NNP-NNP & -0.11 & -0.05 & 0 & -0.38 & -0.54 \\
\hline \multicolumn{6}{|c|}{ TYPES } \\
\hline PTYPE:x:2 & -0.07 & -0.15 & 0.35 & 0.18 & -0.31 \\
\hline CTYPE:XX & -2.02 & 0.46 & 0.19 & 0.57 & 0.80 \\
\hline NTYPE:Xx & -0.22 & -0.42 & -0.19 & 0.29 & 0.54 \\
\hline PTYPE-CTYPE: $x: 2-X x$ & -0.20 & 0.08 & 0.10 & 0.10 & -0.09 \\
\hline CTYPE-NTYPE:Xx-Xx & 0.55 & -0.13 & -0.55 & -0.13 & 0.26 \\
\hline PTYPE-CTYPE-NTYPE: $x: 2-X x-X x$ & 0.10 & 0.37 & 0.10 & 0.12 & -0.69 \\
\hline \multicolumn{6}{|c|}{ WORDS/TYPES } \\
\hline PWORD-CTYPE:at-Xx & -0.21 & 0.57 & -0.21 & 0.41 & -0.56 \\
\hline CTYPE-NWORD:Xx-Road & -0.01 & 0.27 & -0.01 & -0.23 & -0.03 \\
\hline \multicolumn{6}{|c|}{ STATES } \\
\hline PSTATE:O & 2.91 & -0.92 & -0.72 & -0.58 & -0.70 \\
\hline PPSTATE-PSTATE:O-O & 1.14 & -0.60 & -0.08 & -0.43 & -0.04 \\
\hline \multicolumn{6}{|c|}{ WORDS/STATES } \\
\hline PSTATE-CWORD:O-Grace & -0.01 & 0 & 0 & -0.02 & 0.03 \\
\hline \multicolumn{6}{|c|}{ TAGS/STATES } \\
\hline PSTATE-PTAG-CTAG:O-IN-NNP & 0.12 & 0.59 & -0.29 & -0.28 & -0.14 \\
\hline $\begin{array}{l}\text { PPSTATE-PPTAG-PSTATE-PTAG- } \\
\text { CTAG:O-NN-O-IN-NNP }\end{array}$ & 0.01 & -0.03 & -0.31 & 0.31 & 0.01 \\
\hline \multicolumn{6}{|c|}{ TYPES/STATES } \\
\hline PSTATE-CTYPE:O-Xx & -1.13 & 0.37 & -0.12 & 0.20 & 0.68 \\
\hline PSTATE-NTYPE:O-Xx & -0.69 & -0.3 & 0.29 & 0.39 & 0.30 \\
\hline PSTATE-PTYPE-CTYPE:O-x:2-Xx & -0.28 & 0.82 & -0.10 & -0.26 & -0.20 \\
\hline $\begin{array}{l}\text { PPSTATE-PPTYPE-PSTATE- } \\
\text { PTYPE-CTYPE:O-x-O-x:2-Xx } \\
\end{array}$ & -0.22 & -0.04 & -0.04 & -0.06 & 0.22 \\
\hline Total: & -1.40 & 2.68 & -1.74 & -0.19 & -0.58 \\
\hline
\end{tabular}

Table 3: Example of the features and weights at a local decision point: deciding the classification of Grace.

\section{References}

Daniel M. Bikel, Scott Miller, Richard Schwartz, and Ralph Weischedel. 1997. Nymble: a high-performance learning name-finder. In Proceedings of ANLP-97, pages 194-201.

Andrew Borthwick. 1999. A Maximum Entropy Approach to Named Entity Recognition. Ph.D. thesis, New York University.

Silviu Cucerzan and David Yarowsky. 1999. Language independent named entity recognition combining morphological and contextual evidence. In Joint SIGDAT Conference on EMNLP and VLC.

\begin{tabular}{|l|c|c|c|}
\cline { 2 - 4 } \multicolumn{1}{c|}{ English devel. } & Precision & Recall & $\mathrm{F}_{\beta=1}$ \\
\hline LOC & 94.44 & 94.34 & 94.39 \\
MISC & 90.62 & 83.84 & 87.10 \\
ORG & 87.63 & 89.26 & 88.44 \\
PER & 93.86 & 97.01 & 95.41 \\
\hline Overall & 92.15 & 92.39 & 92.27 \\
\hline
\end{tabular}

\begin{tabular}{|l|c|c|c|}
\cline { 2 - 4 } \multicolumn{1}{l|}{ English test } & Precision & Recall & $\mathrm{F}_{\beta=1}$ \\
\hline LOC & 90.04 & 89.93 & 89.98 \\
MISC & 83.49 & 77.07 & 80.15 \\
ORG & 82.49 & 78.57 & 80.48 \\
PER & 86.66 & 95.18 & 90.72 \\
\hline Overall & 86.12 & 86.49 & 86.31 \\
\hline
\end{tabular}

\begin{tabular}{|l|c|c|c|}
\cline { 2 - 4 } \multicolumn{1}{c|}{ German devel. } & Precision & Recall & $\mathrm{F}_{\beta=1}$ \\
\hline LOC & 75.53 & 66.13 & 70.52 \\
MISC & 78.71 & 47.23 & 59.03 \\
ORG & 77.57 & 53.51 & 63.33 \\
PER & 72.36 & 71.02 & 71.69 \\
\hline Overall & 75.36 & 60.36 & 67.03 \\
\hline
\end{tabular}

\begin{tabular}{|l|c|c|c|}
\cline { 2 - 4 } \multicolumn{1}{l|}{ German test } & Precision & Recall & $\mathrm{F}_{\beta=1}$ \\
\hline LOC & 78.01 & 69.57 & 73.54 \\
MISC & 75.90 & 47.01 & 58.06 \\
ORG & 73.26 & 51.75 & 60.65 \\
PER & 87.68 & 79.83 & 83.57 \\
\hline Overall & 80.38 & 65.04 & 71.90 \\
\hline
\end{tabular}

Table 4: Final results obtained for the development and test sets for each language on the shared task.

Shai Fine, Yoram Singer, and Naftali Tishby. 1998. The hierarchical hidden markov model: Analysis and applications. Machine Learning, 32:41-62.

Andrew McCallum, Dayne Freitag, and Fernando Pereira. 2000. Maximum entropy Markov models for information extraction and segmentation. In ICML-2000.

Andrei Mikheev. 1997. Automatic rule induction for unknown-word guessing. Computational Linguistics, 23(3):405-423.

Adwait Ratnaparkhi. 1996. A maximum entropy model for part-of-speech tagging. In EMNLP 1, pages 133142.

Joseph Smarr and Christopher D. Manning. 2002. Classifying unknown proper noun phrases without context. Technical Report dbpubs/2002-46, Stanford University, Stanford, CA.

Nina Wacholder, Yael Ravin, and Misook Choi. 1997. Disambiguation of proper names in text. In ANLP 5, pages 202-208. 